\title{
Dopamine as a potential rescue therapy for refractory protein-losing enteropathy in Fontan-palliated patients
}

\author{
Joshua M. Friedland-Little ${ }^{1}$ (D) | Robert J. Gajarski ${ }^{2}$ | Kurt R. Schumacher ${ }^{3}$
}

${ }^{1}$ Division of Pediatric Cardiology, Seattle Children's Hospital, University of Washington, Seattle, WA, USA

${ }^{2}$ Division of Pediatric Cardiology, Nationwide Children's Hospital, Ohio State University,

Columbus, OH, USA

${ }^{3}$ Division of Pediatric Cardiology, Mott Children's Hospital, University of Michigan, Ann Arbor, MI, USA

\section{Correspondence}

Joshua M. Friedland-Little, Heart Center, Seattle Children's Hospital, Seattle, WA, USA. Email: joshua.friedland-little@seattlechildrens. org

\begin{abstract}
PLE is an important cause of morbidity and mortality in patients who have undergone Fontan palliation. While multiple PLE therapies have been reported, none has proved consistently effective. Patients who do not respond to "standard" PLE therapies face poor long-term outcomes. We report here a significant response to dopamine infusion in three patients with chronic, refractory PLE. We hypothesize that this response may be at least partially due to a dopamine effect on lymphatic receptors rather than to an augmentation of cardiac output.
\end{abstract}

KEYWORDS

dopamine, fontan operation, heart transplantation, protein-losing enteropathy

\section{1 | INTRODUCTION}

PLE is a relatively uncommon but potentially life-threatening complication of Fontan-palliated single ventricle congenital heart disease. PLE is characterized by protein loss through the intestinal lumen with a cascade of downstream effects. ${ }^{1}$ It has been reported in $1 \%-13 \%$ of Fontan patients, ${ }^{2,3}$ although the potential for asymptomatic disease coupled with the lack of routine screening makes the true prevalence unknown. Mortality of up to $50 \%$ has been reported within 5 years of the diagnosis, ${ }^{2,4}$ and although survival has likely improved in the current era, ${ }^{5}$ PLE-associated morbidity remains significant with altered calcium metabolism, growth failure, frequent hospitalizations, and increased infection risk related to immune dysfunction common among PLE patients. ${ }^{6}$ Although multiple therapies have been used with apparent success in select patients, no therapy short of heart transplant has proven to consistently alleviate PLE symptoms. Here, we report a significant response to dopamine infusion in three patients with chronic, severe PLE unresponsive to standard therapies and discuss potential physiologic mechanisms for this observed response.

\section{2 | CASE REPORT}

Patient 1 was a 19-year-old man with an unbalanced (right dominant) atrioventricular septal defect status-post fenestrated lateral tunnel

Abbreviations: bpm, beats per minute; PLE, protein-losing enteropathy
Fontan at $1 \frac{1}{2}$ years of age. Despite good Fontan hemodynamics, at age 9 he developed symptomatic PLE, with hypoalbuminemia and elevated fecal $\alpha-1$ antitrypsin. He was treated with therapy including budesonide, sildenafil, spironolactone, and octreotide and for many years required one to two annual admissions for albumin infusion. At age 17, his symptoms worsened dramatically with marked fluid overload and persistent hypoalbuminemia. Cardiac catheterization revealed a Fontan pressure of $13 \mathrm{~mm} \mathrm{Hg}$, right ventricular end-diastolic pressure of $8 \mathrm{~mm} \mathrm{Hg}$, a cardiac index of $3.9 \mathrm{~L} / \mathrm{min} / \mathrm{m}^{2}$, systemic vascular resistance of $12.5 \mathrm{~mm} \mathrm{Hg} / \mathrm{L} / \mathrm{min}$, and pulmonary vascular resistance of 1.5 Wood units $\times \mathrm{m}^{2}$. His echocardiogram demonstrated mildly depressed right ventricular systolic function and mild tricuspid regurgitation. Over the next 2 years, he required 17 admissions for albumin replacement. He developed acute kidney injury during one hospitalization, and milrinone was initiated. This resulted in acute hypotension, and the milrinone was stopped and replaced with dopamine. On dopamine, his kidney injury improved, and his albumin level was noted to rapidly stabilize. Of note, there was no apparent change in resting heart rate on dopamine, with documented heart rates ranging between 80 and 120 bpm before and after dopamine was initiated. The patient was discharged home on dopamine and required only one admission for PLE (1 month after starting dopamine) over the subsequent 19 months prior to heart transplantation.

Patient 2 was a 21-year-old man with hypoplastic left heart syndrome status post-fenestrated lateral tunnel Fontan at 2 years of age. He was diagnosed with PLE at age 13 , with hypoalbuminemia and elevated fecal $\alpha-1$ antitrypsin. He was treated with oral budesonide, 
sildenafil, heparin, and octreotide and required one to three admissions for albumin replacement per year over the next several years. At age 18, his symptoms worsened significantly, with marked fluid overload and refractory hypoalbuminemia requiring ongoing outpatient albumin infusions three to four times per week. Cardiac catheterization revealed a mean Fontan pressure of $12 \mathrm{~mm} \mathrm{Hg}$, pulmonary capillary wedge pressure of $7 \mathrm{~mm} \mathrm{Hg}$, a cardiac index of $3.2 \mathrm{~L} / \mathrm{min} / \mathrm{m}^{2}$, a systemic vascular resistance of $20 \mathrm{~mm} \mathrm{Hg} / \mathrm{L} / \mathrm{min}$, and a pulmonary vascular resistance of 1.8 Wood units $\times \mathrm{m}^{2}$. His echocardiogram revealed normal right ventricular systolic function and mild tricuspid regurgitation. Right ventricular systolic function was also normal as assessed by cardiac MRI, with an ejection fraction of $55 \%$. Dopamine was initiated at $5 \mu \mathrm{g} / \mathrm{kg} / \mathrm{min}$ and continued in an outpatient setting. Heart rate did not change on dopamine, ranging between 70 and $110 \mathrm{bpm}$ pre-dopamine and between 60 and $100 \mathrm{bpm}$ on dopamine. Within 2 months of dopamine initiation, he had progressively weaned to weekly albumin infusions. At the time of transplant, he had weaned further and maintained a serum albumin greater than $3.5 \mathrm{mg} / \mathrm{dL}$ for 9 days without supplementation for the first time in more than 3 years.

Patient 3 was a 15-year-old female with hypoplastic left heart syndrome status post-lateral tunnel fenestrated Fontan at 23 months of age. She was diagnosed with PLE at age 5 and initially responded to diuretic therapy and later oral budesonide, but starting at age 12 she required increasingly frequent admissions for fluid overload and albumin supplementation. She persistently had serum albumin levels between 2 and $2.5 \mathrm{mg} / \mathrm{dL}$ as well as chronic ascites, and she was treated with albumin infusions 2-4 times per month as symptoms dictated. Cardiac catheterization revealed a Fontan pressure of $17 \mathrm{~mm} \mathrm{Hg}$, a right ventricle end-diastolic pressure of $13 \mathrm{~mm} \mathrm{Hg}$, a cardiac index of $2.9 \mathrm{~L} / \mathrm{min} / \mathrm{m}^{2}$, systemic vascular resistance of $18 \mathrm{~mm} \mathrm{Hg} / \mathrm{L} / \mathrm{min}$, and a pulmonary vascular resistance of 0.8 Wood units $\times \mathrm{m}^{2}$. Echocardiogram demonstrated normal right ventricular systolic function and mildmoderate tricuspid insufficiency. Dopamine at $5 \mu \mathrm{g} / \mathrm{kg} / \mathrm{min}$ was initiated at transplant listing. As with the other patients, there was no significant change in heart rate, which ranged between 60 and 90 bpm before and after dopamine was started. Within 2 weeks, serum albumin levels increased and remained $>3 \mathrm{mg} / \mathrm{dL}$ without supplementation and her ascites resolved. After approximately 3 months, the patient and family elected not to pursue heart transplantation, so dopamine was weaned off. Within 2 weeks of stopping dopamine, her serum albumin level fell to $2 \mathrm{mg} / \mathrm{dL}$ with an accompanying $2 \mathrm{~kg}$ weight gain and return of significant ascites.

\section{DISCUSSION}

In this report, we describe three patients with chronic, severe, and previously refractory PLE who each demonstrated rapid improvement in PLE symptoms and serum albumin levels after the initiation of continuous dopamine. In seeking to explain this response, the initial assumption may be that dopamine's inotropic effects improved systemic ventricular systolic function and thereby augmented cardiac output. Although the pathophysiology of PLE remains unclear, there is a body of evidence suggesting that interventions which improve cardiac output can in some cases improve PLE symptoms. ${ }^{1}$ The patients in our series, however, had normal or only mildly depressed systemic ventricular systolic function as assessed by echocardiogram. While echocardiogram is an imperfect tool to assess systolic function in a systemic right ventricle, all patients had normal resting cardiac output measured in the catheterization laboratory, and the one patient for whom MRI data were available had normal systolic function as assessed by this modality as well. The degree of improvement in PLE symptoms in the patients in this series seems disproportionate to a potential augmentation of systolic function assessed as normal or only mildly depressed by the modalities available.

There are other potential effects of dopamine in addition to improved contractile function. Dopamine also has chronotropic effects, and there is evidence that atrial pacing can improve PLE symptoms in patients with sinus node dysfunction. ${ }^{7}$ None of the patients had significant resting chronotropic incompetence, however, and no changes in heart rate were documented after dopamine was initiated. Another hypothesis is that dopamine increased mesenteric blood flow by decreasing mesenteric vascular resistance. There is evidence that mesenteric vascular resistance is abnormally elevated in Fontan patients with PLE, 8,9 and decreased mesenteric perfusion may play a role in PLE pathophysiology. Dopamine, particularly at low doses, does decrease mesenteric vascular resistance, and an augmentation of mesenteric blood flow may have contributed to the therapeutic response seen.

Myocardial function and cardiac output of the patients in this series were only assessed at rest, and it is well documented that Fontan patients have reduced exercise capacity and limited ability to augment their cardiac output with exertion. ${ }^{10,11}$ While much of this limitation is thought to be secondary to an inability to effectively increase preload in the setting of increased metabolic demands, the inotropic and chronotropic effects of dopamine may have improved the ability of the patients described here to increase their cardiac output with exercise, and this may also have contributed to some improvement in PLE symptoms.

One intriguing alternative hypothesis to explain the improvement seen in these patients is that dopamine served to augment lymphatic circulation. Recent data suggest dysregulation of lymphatic circulation can contribute to the development of PLE in Fontan patients, and mechanical blockage of lymphatic drainage can be identified in significant numbers of Fontan patients with PLE. ${ }^{12}$ Surgical decompression of the thoracic duct has been shown to relieve symptoms in two Fontan patients with otherwise refractory PLE. ${ }^{13}$ Further evidence supporting a link between disrupted lymph circulation and PLE in Fontan patients is the observation that Fontan patients with PLE display immune abnormalities which are also found in patients with PLE secondary to intestinal lymphangiectasia, but not in patients with PLE related to ulcerative colitis or celiac disease. ${ }^{14}$

Normal lymphatic circulation relies on a series of lymph pumps and one-way valves to transport lymph fluid from the periphery to the thoracic and lymphatic ducts and then into the central venous system. This system is in effect a parallel circulatory system, with the 
intrinsic lymph pumps functioning as a cardiac equivalent, providing contractile force for forward lymph flow. ${ }^{15}$ Like the cardiovascular system, the lymphatic system is subject to complex neurohormonal regulation that influences both pump contractility and lymph vessel compliance. Interestingly, lymph vessels and lymph pumps display a relationship to lymphatic pressure and vessel wall stretch that is analogous to the Frank-Starling relationship between preload and contractility. Increased lymph pressure/stretch (ie, preload) increases lymph pump contractile strength up to a point, but beyond that point increased pressure/stretch results in decreased contractile strength. ${ }^{15}$ The threshold at which this shift occurs varies depending on species and physical tissue characteristics, but generally is low. One may speculate that the persistently elevated systemic venous pressures of Fontan patients may push the "lymphatic Starling curve" too far in some patients and lead to decreased contractile strength of the intrinsic lymph pumps.

In another analogy to the cardiovascular system, the contractility of lymphatic vessels can be augmented by inotropic agents, including dopamine. ${ }^{15,16}$ Dopamine has been shown to cause prenodal lymphatic constriction in canine models, an effect mediated by $\alpha$-adrenergic receptors. ${ }^{17}$ While the effect of dopamine on human lymphatic contractility is not known, there is evidence that the human thoracic duct has significant adrenergic innervation. ${ }^{18}$ Although the potential effect of dopamine on the lymphatic circulation of patients in this series remains speculative, presumed dysfunctional lymphatic circulation in Fontan patients with PLE may serve as a target for pharmacologic intervention and direct new potential avenues for research.

In conclusion, chronic dopamine infusion may be an effective therapy for refractory PLE in some patients. The mechanism by which dopamine may improve PLE is unclear, and further study is needed to confirm the potential therapeutic benefit of dopamine as an additional treatment modality for PLE as well as to clarify its mechanism of action in this setting.

\section{AUTHORS' CONTRIBUTIONS}

Joshua M. Friedland-Little, Robert J. Gajarski, and Kurt R. Schumacher: Participated in data analysis and interpretation, manuscript drafting and revision, and approval of the submitted manuscript.

\section{REFERENCES}

1. Rychik J. Protein-losing enteropathy after Fontan operation. Congenit Heart Dis. 2007;2:288-300.

2. Feldt RH, Driscoll DJ, Offord KP, et al. Protein-losing enteropathy after the Fontan operation. J Thorac Cardiovasc Surg. 1996;112:672-680.
3. Stamm C, Friehs I, Mayer JE Jr, et al. Long-term results of the lateral tunnel Fontan operation. J Thorac Cardiovasc Surg. 2001;121:28-41.

4. Mertens L, Hagler DJ, Sauer U, Somerville J, Gewillig M. Proteinlosing enteropathy after the Fontan operation: an international multicenter study. PLE study group. J Thorac Cardiovasc Surg. 1998;115:1063-1073.

5. John AS, Johnson JA, Khan M, Driscoll DJ, Warnes CA, Cetta F. Clinical outcomes and improved survival in patients with protein-losing enteropathy after the Fontan operation. J Am Coll Cardiol. 2014;64:54-62.

6. Schumacher KR, Stringer KA, Donohue JE, et al. Fontan-associated protein-losing enteropathy and plastic bronchitis. I Pediatr. 2015;166:970-977.

7. Cohen MI, Rhodes LA, Wernovsky G, Gaynor JW, Spray TL, Rychik J. Atrial pacing: an alternative treatment for protein-losing enteropathy after the Fontan operation. J Thorac Cardiovasc Surg. 2001;121:582-583.

8. Rychik J, Gui-Yang S. Relation of mesenteric vascular resistance after Fontan operation and protein-losing enteropathy. Am J Cardiol. 2002;90:672-674.

9. Ostrow AM, Freeze H, Rychik J. Protein-losing enteropathy after Fontan operation: investigations into possible pathophysiologic mechanisms. Ann Thorac Surg. 2006;82:695-700.

10. Paridon SM, Mitchell PD, Colan SD, et al. A cross-sectional study of exercise performance during the first 2 decades of life after the Fontan operation. J Am Coll Cardiol. 2008;52:99-107.

11. Goldberg DJ, French B, McBride MG, et al. Impact of oral sildenafil on exercise performance in children and young adults after the fontan operation: a randomized, double-blind, placebo-controlled, crossover trial. Circulation. 2011;123:1185-1193.

12. Meadows J, Gauvreau K, Jenkins K. Lymphatic obstruction and protein-losing enteropathy in patients with congenital heart disease. Congenit Heart Dis. 2008;3:269-276.

13. Antonio M, Gordo A, Pereira C, Pinto F, Fragata I, Fragata J. Thoracic duct decompression for protein-losing enteropathy in failing Fontan circulation. Ann Thorac Surg. 2016;101:2370-2373.

14. Magdo HS, Stillwell TL, Greenhawt MJ, et al. Immune abnormalities in fontan protein-losing enteropathy: a case-control study. J Pediatr. 2015;167:331-337.

15. Zawieja DC. Contractile physiology of lymphatics. Lymphat Res Biol. 2009;7:87-96.

16. Ohhashi T, Kawai Y, Azuma T. The response of lymphatic smooth muscles to vasoactive substances. Pflugers Arch. 1978;375:183-188.

17. Dobbins DE. Receptor mechanisms of prenodal lymphatic constriction by dopamine. Regul Pept. 2003;114:7-13.

18. Telinius N, Baandrup U, Rumessen J, et al. The human thoracic duct is functionally innervated by adrenergic nerves. Am J Physiol Heart Circ Physiol. 2014;306:H206-H213.

How to cite this article: Friedland-Little JM, Gajarski RJ, Schumacher KR. Dopamine as a potential rescue therapy for refractory protein-losing enteropathy in Fontan-palliated patients. Pediatr Transplant. 2017;21:e12925.

https://doi.org/10.1111/petr.12925 